\title{
285 BREAKING THROUGH THE RESISTANCE OF BREAST CANCER TO IMMUNE CHECKPOINT BLOCKERS IN A UNIQUE MOUSE MODEL OF HR+ DISEASE
}

${ }^{1}$ Norma Bloy*, ${ }^{2}$ Aitziber Buqué Martinez, ${ }^{2}$ Bhavneet Binder, ${ }^{2}$ Giulia Petroni, ${ }^{2}$ Takahiro Yamazaki, ${ }^{2} \mathrm{Ai}$ Sato, ${ }^{2}$ Olivier Elemento, ${ }^{2}$ Silvia Formenti, ${ }^{2}$ Lorenzo Galluzzi. ' Weill Cornell Medical College, New York, NY, USA; ${ }^{2}$ Weill Cornell Medicine, New York, NY, USA

Background Hormone receptor (HR)+ breast cancer (BC) causes most BC-related deaths in the US. ${ }^{1}$ Standard treatment for non-metastatic disease involves surgery plus adjuvant hormonotherapy. However, approximately $50 \%$ of patients ultimately relapse and require additional lines of treatment including chemotherapy, which is unfortunately associated with limited clinical benefits and severe toxicity. In $\mathrm{HR}+\mathrm{BC}$ patients, the efficacy of immunotherapy has also been disappointing so far. Indeed, objective responses to PD-1 blockade with pembrolizumab in women with $\mathrm{HR}+\mathrm{BC}$ have been in the range of 5-10\%, with no clear advantage on survival. Thus, resistance to PD-1 blockers constitutes a major obstacle towards the implementation of immunotherapy in $\mathrm{HR}+\mathrm{BC}$ patients.

Methods To obtain insights into the immunological alterations accompanying disease relapse in $\mathrm{HR}+\mathrm{BC}$ exposed to $\mathrm{PD}-1$ blockade, we harnessed a unique endogenous model of $\mathrm{BC}$ driven in immunocompetent mice by progesterone and a carcinogen. This model recapitulates key aspects of human luminal B BC, including a relatively 'cold' microenvironment, hence limited sensitivity to PD-1 blockade. ${ }^{2}$ We undertook an indepth characterization of the tumors (by DNAseq and RNAseq) and systemic (by flow cytometry on the splenic compartment) immune microenvironment of $\mathrm{C} 57 \mathrm{BL} / 6$ female mice bearing tumors that recovered normal growth after PD-1 treatment.

Results There was no clear difference after PD-1 blockade at the systemic level in the myeloid, or lymphoid compartments; or in the activation of $\mathrm{T}$ cells, nor their capacity to degranulate upon ex vivo stimulation. Whole exome sequencing showed a higher mutational burden in PD-1 treated tumors after relapse. At the gene expression level, unsupervised analysis showed a clustering independent of the treatment groups, probably due to the heterogeneity of the model. Targeted pathway analysis with supervised clustering showed however differences in immune pathways that are currently further investigated, and results will be available shortly.

Conclusions Breaking through resistance of $\mathrm{HR}+$ tumors to PD-1 blockers can direct strategies to overcome resistance in $\mathrm{HR}+\mathrm{BC}$ patients, the majority of BC patients. If successful, this can inform therapeutic approaches to enable superior therapeutic responses in patients with $\mathrm{HR}+\mathrm{BC}$, hence significantly reducing $\mathrm{BC}$-related deaths.

\section{REFERENCES}

1.. Siegel RL, Miller KD, Jemal A. Cancer statistics, 2020. CA Cancer J Clin 2020;70:7-30.

2. Buque A, Bloy N, Perez-Lanzon M, Iribarren K, Humeau J, Pol JG, Levesque S, Mondragon L, Yamazaki T, Sato A, Aranda F, Durand S, Boissonnas A, Fucikova J, Senovilla L, Enot D, Hensler M, Kremer M, Stoll G, Hu Y, Massa C, Formenti SC, Seliger B, Elemento O, Spisek R, Andre F, Zitvogel L, Delaloge S, Kroemer G, Galluzzi L. Immunoprophylactic and immunotherapeutic control of hormone receptor-positive breast cancer. Nat Commun 2020;11:3819.

Ethics Approval This study was approved by Weill Cornell Medical College's Ethics Board; approval number 2018-0053.

http://dx.doi.org/10.1136/jitc-2021-SITC2021.285 